\title{
SUPERSYMMETRIC TECHNIQUES APPLIED TO THE JACOBI EQUATION
}

Haret C. Rosu [ and Juan R. Guzmán

Instituto de Física - IFUG, Apdo Postal E-143, León, Gto, Mexico

\begin{abstract}
The simple supersymmetric approach recently used by Dutt, Gangopadhyaya, and Sukhatme for spherical harmonics is generalized to Jacobi equation, including also the intermediate Gegenbauer case.

Resumen. Un procedimiento supersimétrico simple recientemente usado por Dutt, Gangopadhyaya y Sukhatme para los harmonicos esféricos, es generalizado a la ecuacion de Jacobi, incluyendo tambien el caso intermedio de Gegenbauer.
\end{abstract}

European J. Phys. 19 (May 1998) 287-292 physics/9701016

${ }^{1}$ e-mail: rosu@ifug3.ugto.mx 


\section{INTRODUCTION}

During 1971-1974, supersymmetry (SUSY), a more general (graded) Lie algebraic realization of symmetries, involving combinations of commutation and anti-commutation relations has been discovered as a sort of necessary unifying algebraic ingredient of microscopic bosonic and fermionic degrees of freedom [1]. In 1981, Witten [2] introduced the simplest SUSY realization within nonrelativistic quantum mechanics (SUSYQM) as a toy tool for studying SUSY breaking in quantum field theories. SUSYQM may be seen as a special property of the factorizations of the one-dimensional (1D) Hamiltonians. Namely, one can find a pair of Hamiltonians (usually called bosonic and fermionic partners) having the potentials connected by a single Riccati solution, known as the superpotential. Indeed, if one fixes to zero the ground state energy, one can easily factorize any 1D Hamiltonian as follows

$$
H_{1} \equiv-c^{2} \frac{d^{2}}{d x^{2}}+V_{1}=A^{\dagger} A
$$

where $A^{\dagger}=-c \frac{d}{d x}+W(x), A=c \frac{d}{d x}+W(x)$ and $c=\frac{\hbar}{\sqrt{2 m}}$ for nonrelativistic quantum mechanics. The $W$ function occurring in the factorizing operators is the solution (superpotential) of the following Riccati equation

$$
V_{1}=W^{2}-c W^{\prime} .
$$

The SUSYQM scheme, as initiated by Witten, means to construct the "fermionic" Hamiltonian obtained by reversing the order of the factoring operators, which reads

$$
H_{2}=A A^{\dagger} \equiv-c^{2} \frac{d^{2}}{d x^{2}}+V_{2}
$$

where

$$
V_{2}=W^{2}+c W^{\prime} .
$$

One can show that the energy eigenvalues, the wave functions, the $S$ matrices, the reflection and transmission coefficients of the supersymmetric partners $H_{1}$ and $H_{2}$ are related in a simple manner through the application of the factorizing operators 沺. In particular, the energy spectra are identical except perhaps for the bosonic ground state (for the so-called unbroken SUSY case). In 1983, Gendenshtein [3] noticed that in many important problems there is a very useful relationship between the SUSY partner potentials $V_{1,2}$, known as shape invariance (SI). He showed that if the superpartners are similar in shape and differ only in the parameters that appear in them, i.e., in mathematical terms, if one writes down a SI condition of the form

$$
V_{2}\left(x ; a_{1}\right)=V_{1}\left(x ; a_{2}\right)+R\left(a_{1}\right),
$$

where $a_{1}$ is a set of parameters, and $a_{2}$ is a function of $a_{1}$, such that the remainder $R\left(a_{1}\right)$ is independent of $x$, one can easily derive the energy eigenvalues and eigenfunctions of any potential fulfilling the SI condition. Thus, SI is a valuable parametric connection within SUSYQM.

The coordinate transformation procedure is well known in the framework of SUSYQM (see section 5 in (4⿴囗十). Essentially, one starts with a 1D Schrödinger equation and tries to obtain a better known one by performing a coordinate transformation. This may be considered as a particular case of various transformations of homogeneous linear differential equations of second order involving either the dependent variable, or the independent one, or both, which are recognized as basic methods in the mathematical literature; the reader is directed to the textbooks of Szegö and Zwillinger [5]. However, using SUSYQM, one can exploit further SUSY symmetries, like the aforementioned SI, to get some properties of special functions in an illuminating way. This is precisely the case of a recent paper of Dutt, Gangopadhyaya and Sukhatme (DGS) [6], where they used the change of the independent variable, to recast the associated Legendre equation in the celebrated Schrödinger equation with the - sech potential, which enjoys the SI property. Next, they put to work this concept to derive some known features of spherical harmonics in a simple way.

In the following, after briefly repeating the DGS case of spherical harmonics, we generalize their scheme to the Jacobi equation, presenting also the intermediate Gegenbauer case. 


\section{SPHERICAL HARMONICS}

The equation for the associated Legendre polynomials

$$
\frac{d^{2} y}{d \theta^{2}}+\cot \theta \frac{d y}{d \theta}+\left[l(l+1)-\frac{m^{2}}{\sin ^{2} \theta}\right] y=0
$$

can be transformed into a Schrödinger eigenvalue equation by a mapping function $\theta=f(z)$ that can be found from the condition of putting to nought the coefficient of the first derivative. The result is $\theta \equiv f=$ $2 \arctan \left(e^{z}\right)$. This mapping is equivalent to the replacement $\sin \theta=\operatorname{sech} z$ and $\cos \theta=-\tanh z$. The range of the variable $z$ is the full real line $-\infty<z<\infty$. We notice that $\theta(z)=\frac{\pi}{2}+\operatorname{gd}(z)$, where $\operatorname{gd}(z)$ is the so-called Gudermannian or hyperbolic amplitude function [7. The associated Legendre equation is transformed in one of the best known exactly solvable, SI Schrödinger equation

$$
-\frac{d^{2} v}{d z^{2}}-\left[l(l+1) \operatorname{sech}^{2} z\right] v=-m^{2} v
$$

for which the algebraic SUSY scheme can be readily applied. Since $l$ is always an integer for the common spherical harmonics, Eq. (7) is moreover a reflectionless one (i.e., the calculated reflection coefficient is zero). The energy eigenvalues are known to be of the type $E_{n}=-(l-n)^{2}$, with $n=0,1,2 \ldots N$, where $N$ is the number of bound states the $-l(l+1) \operatorname{sech}^{2}$ potential well can hold, which for spherical harmonics is equal to $l$. Since $E_{n}=-m^{2}$, one gets $n=l-m$. The eigenfunctions $\left|v_{n}(z ; 0)\right\rangle$ can be obtained by repeated application of the creation operators $A^{\dagger}(z ; k) \equiv\left(-\frac{d}{d z}+k \tanh z\right), k \in[0, m-1]$, as follows

$$
\left|v_{n}(z ; 0)\right\rangle \propto\left[\prod_{k=m-1}^{k=0} A^{\dagger}(z ; k)\right]\left|v_{0}(z ; m)\right\rangle,
$$

where the ground state wavefunction is $\left|v_{0}(z ; m)\right\rangle=\operatorname{sech}^{m} z$. The connection between the $v$ functions and the associated Legendre polynomials is given by $P_{l}^{m}(\tanh z) \approx v_{l-m}(z ; l)$.

We notice that the mapping $f$ and the Schrödinger equation (7) were already known to Infeld and Hull [8], who used them in their factorization method.

\section{JACOBI POLYNOMIALS}

A more complicated case is that of Jacobi polynomials which fulfill the differential equation

$$
\left(1-x^{2}\right) y^{\prime \prime}+[\beta-\alpha-(\alpha+\beta+2) x] y^{\prime}+n(n+\alpha+\beta+1) y=0 .
$$

By employing the notations $2(m+1)=\alpha+\beta+2, n(n+\alpha+\beta+1)=\left(n^{\prime}-m\right)\left(n^{\prime}+m+1\right), \beta-\alpha=\gamma$. Eq. (9) turns into

$$
\left(1-x^{2}\right) y^{\prime \prime}+\gamma y^{\prime}-2(m+1) x y^{\prime}+\left(n^{\prime}-m\right)\left(n^{\prime}+m+1\right) y=0
$$

and with $v=\left(1-x^{2}\right)^{m / 2} y$ one gets

$$
\left(1-x^{2}\right) v^{\prime \prime}-(2 x-\gamma) v^{\prime}+\left[n^{\prime}\left(n^{\prime}+1\right)-\frac{m^{2}-\gamma m x}{1-x^{2}}\right] v=0,
$$

or in spherical polar coordinates

$$
\frac{d^{2} v}{d \theta^{2}}+\left(\cot \theta-\frac{\gamma}{\sin \theta}\right) \frac{d v}{d \theta}+\left[n^{\prime}\left(n^{\prime}+1\right)-\frac{m^{2}-\gamma m \cos \theta}{\sin ^{2} \theta}\right] v=0 .
$$

We already see that Eq. (12) presents deviations from the standard associated form. With the change of variable $\theta=f(z)$ and the notations $P(f)$ and $Q(f)$ for the coefficients of the first derivative of $v$ and of $v$, respectively, in Eq. (12), one can get the following form

$$
\frac{d^{2} v}{d z^{2}}+\left[f^{\prime} P(f)-\frac{f^{\prime \prime}}{f^{\prime}}\right] \frac{d v}{d z}+Q(f) f^{\prime 2} v=0 .
$$


Again the coefficient of the first derivative is put to nought, leading to the differential equation

$$
\frac{f^{\prime \prime}}{f^{\prime}}=f^{\prime} P(f)
$$

which can be solved by the substitution $u=f^{\prime} \equiv \frac{d f}{d z}$ leading to

$$
\frac{d u}{d f}=u P(f)
$$

with the solution $v=e^{\int P(f) d f}$. Since $\int P(\theta) d \theta=\ln \left[\frac{\sin \theta}{\tan \gamma(\theta / 2)}\right]$ we have $\frac{d \theta}{d z}=\frac{\sin \theta}{\tan ^{\gamma}(\theta / 2)}$. Thence, $z=$ $\int \tan ^{\gamma}(\theta / 2) \csc \theta d \theta+$ const. We have found that a convenient choice for the constant of integration is const $=$ $-\frac{1}{\gamma}$. Using $g=\tan (\theta / 2)$, one gets $z=\int g^{\gamma-1} d g-\frac{1}{\gamma}$. Thus,

$$
z= \begin{cases}\frac{\tan ^{\gamma}(\theta / 2)}{\gamma}-\frac{1}{\gamma} & \gamma \neq 0 \\ \ln [\tan (\theta / 2)] & \gamma=0\end{cases}
$$

The angular variable can be written as

$$
\theta= \begin{cases}2 \arctan \left[(\gamma z+1)^{1 / \gamma}\right] & \gamma \neq 0 \\ 2 \arctan \left(e^{z}\right) & \gamma=0\end{cases}
$$

One can show that the mapping $f$ for $\gamma \neq 0$ is equivalent to the replacements $\sin \theta=\frac{2}{(\gamma z+1)^{-1 / \gamma}+(\gamma z+1)^{1 / \gamma}}=$ $\operatorname{sech} w$ and $\cos \theta=\frac{(\gamma z+1)^{-1 / \gamma}-(\gamma z+1)^{1 / \gamma}}{(\gamma z+1)^{-1 / \gamma}+(\gamma z+1)^{1 / \gamma}}=-\tanh w$, where $w=\frac{\ln (\gamma z+1)}{\gamma}$. For the general case $\gamma \neq 0$ one can get an equation of the form

$$
-\frac{d^{2} v}{d z^{2}}-\left[n^{\prime}\left(n^{\prime}+1\right) \frac{\operatorname{sech}^{2}\left(\frac{\ln (\gamma z+1)}{\gamma}\right)}{(\gamma z+1)^{2}}+\frac{m^{2}+\gamma m \tanh \left(\frac{\ln (\gamma z+1)}{\gamma}\right)}{(\gamma z+1)^{2}}\right] v=0 .
$$

It can be interpreted as a Schrödinger equation at zero energy. One set of solutions $v$ are of the type $v_{n}=\left[1-\tanh ^{2}\left(\frac{\ln (\gamma z+1)}{\gamma}\right)\right]^{\frac{\alpha+\beta}{4}} P_{n}^{\alpha, \beta}\left(\tanh \left(\frac{\ln (\gamma z+1)}{\gamma}\right)\right)$, where $P_{n}^{\alpha, \beta}$ are Jacobi polynomials.

Up to now we did not use the $\alpha, \beta$ asymmetry in the function $v$. If we do that by using $v=(1-x)^{\alpha / 2}(1+$ $x)^{\beta / 2} y$ in Eq. (10), one gets

$$
\left(1-x^{2}\right) v^{\prime \prime}-2 x v^{\prime}+\left[n^{\prime}\left(n^{\prime}+1\right)-\frac{\delta+\epsilon x}{1-x^{2}}\right] v=0,
$$

where $\delta=m(2 m+1)+\gamma^{2}$ and $\epsilon=-\gamma(3 m+1)$. In spherical coordinates $(x=\cos \theta)$ Eq. (17) reads

$$
\frac{d^{2} v}{d \theta^{2}}+\cot \theta \frac{d v}{d \theta}+\left[n^{\prime}\left(n^{\prime}+1\right)-\frac{\delta+\epsilon \cos \theta}{\sin ^{2} \theta}\right] v=0
$$

which though not in the standard associated Legendre form has the advantage that the change of variable $\theta=2 \arctan \left(e^{z}\right)$ can be used to get

$$
\frac{d^{2} v}{d z^{2}}+\left[n^{\prime}\left(n^{\prime}+1\right) \operatorname{sech}^{2} z+\epsilon \tanh z\right] v=\delta v
$$

A simplified form containing only the tanh function is the following [9]

$$
\frac{d^{2} v}{d z^{2}}+\left[A \tanh ^{2} z+2 B \tanh z\right] v=C v
$$

where $A=-n^{\prime}\left(n^{\prime}+1\right), B=-\gamma \frac{(3 m+1)}{2}, C=-n^{\prime}\left(n^{\prime}+1\right)+m(2 m+1)+\gamma^{2}$, and it is supposed that $B<n^{\prime 2}$ [9]. As discussed by DGS and by other authors [10] such a Schrödinger equation corresponds to the motion of an electron in a Coulomb field in the presence of an Aharonov-Bohm potential and a Dirac 
monopole potential. It is also a Rosen-Morse II problem which is known to be shape invariant [6]. The energy eigenvalues and eigenfunctions are in our notations as follows [9]

$$
\begin{gathered}
E_{n}=n^{\prime}\left(n^{\prime}+1\right)-\left(n^{\prime}-n\right)^{2}-\frac{B^{2}}{\left(n^{\prime}-n\right)^{2}}, \\
v_{n}=(\sqrt{1-\tanh z})^{n^{\prime}-n+r}(\sqrt{1+\tanh z})^{n^{\prime}-n-r} P_{n}^{n^{\prime}-n+r, n^{\prime}-n-r}(\tanh z),
\end{gathered}
$$

where $r=\frac{B}{n^{\prime}-n}$.

\section{GEGENBAUER POLYNOMIALS}

The Gegenbauer polynomials $C_{p}^{q}(x)$ are polynomial solutions of the ultraspherical equation

$$
\left(1-x^{2}\right) y^{\prime \prime}-(2 q+1) x y^{\prime}+p(p+2 q) y=0 .
$$

The ultraspherical case can be considered as the particular $\gamma=0$ Jacobi case in the formulas 9-16 of the previous section, and at the same time as a slightly generalized spherical harmonics case of the first section. One gets immediately the shape invariant, exactly solvable Schrödinger equation

$$
-\frac{d^{2} v}{d z^{2}}-\left[n^{\prime}\left(n^{\prime}+1\right) \operatorname{sech}^{2} z\right] v=-m^{\prime 2} v
$$

where $n^{\prime}=p+q-\frac{1}{2}$ and $m^{\prime}=q-\frac{1}{2}$. For a full equivalence to Eqs. (6) and (7) $q$ should be half integer. The energy eigenvalues of the potential $V(z)=-n^{\prime}\left(n^{\prime}+1\right) \operatorname{sech}^{2} z$ are $E_{n}=-\left(n^{\prime}-n\right)^{2},(n=0,1,2, \ldots N)$, where $N$ is the number of bound states in the potential well, and is equal to the largest integer contained in $n^{\prime}$. The eigenfunctions $v_{n}\left(z ; n^{\prime}\right)$ are obtained by applying the factorization (creation) operators $A^{\dagger}\left(z ; a_{i}\right)=$ $\left(-\frac{d}{d z}+a_{i} \tanh z\right)$, where $a_{i}=n^{\prime}-i$, onto the ground state wave function $v_{0}\left(z ; a_{n}\right)=\operatorname{sech}^{n^{\prime}-n} z \equiv \operatorname{sech}^{q-\frac{1}{2}} z$, i.e.,

$$
v_{n}\left(z ; n^{\prime}\right) \approx A^{\dagger}\left(z ; n^{\prime}\right) A^{\dagger}\left(z ; n^{\prime}-1\right) A^{\dagger}\left(z ; n^{\prime}-2\right) \ldots A^{\dagger}\left(z ; n^{\prime}-n+1\right) \operatorname{sech}^{n^{\prime}-n} z
$$

which is an analog of Eq. (8). In the case of this section $n=n^{\prime}-m^{\prime} \equiv p$, and therefore we are dealing with associated Legendre functions of the type $v_{n} \equiv P_{n^{\prime}}^{m^{\prime}}(\tanh z)$. The original Gegenbauer polynomials can be

written formally as $C_{p}^{q}(x) \propto\left(1-\tanh ^{2} z\right)^{\frac{1-2 q}{4}} P_{p+q-\frac{1}{2}}^{q-\frac{1}{2}}(\tanh z)$; the proportionality is used since we are not addressing the normalization issue here. Although this relationship appears to be known, see [11], we found it in a simple way. The $n^{\prime}\left(n^{\prime}+1\right) \operatorname{sech}^{2} z$ potential is reflectionless if and only if $n^{\prime}$ is an integer. Since $p$ should be an integer, the reflectionless property requires again a half integer $q$.

Gegenbauer polynomials are connecting higher-dimensional spherical harmonics to the usual twodimensional ones. In the theory of hyperspherical harmonics they play a role which is analogous to the role played by Legendre polynomials in the usual three-dimensional space [12]. Therefore, we believe our discussion to be useful in such a context as well.

\section{ACKNOWLEDGMENT}

The work was supported in part by the CONACyT Project No. 4868-E9406 and a CONACyT undergraduate fellowship. The authors thank the referee for suggestions.

\section{References}

[1] Gel'fand Y A and Likhtman E P 1971 JETP Lett. 13 323; Ramond P 1971 Phys. Rev. D 3 2415; Neveu A and Schwarz J 1971 Nucl. Phys. B 31 86; Volkov D and Akulov V 1973 Phys. Lett. B 46 109; Wess J and Zumino B 1974 Nucl. Phys. B 7039 and ibid. B 781

[2] Witten E 1981 Nucl. Phys. B 188513

[3] Gendenshtein L 1983 Pis'ma Zh. Eksp. Teor. Fiz. 38299 [JETP Lett. 38 356] 
[4] Cooper F, Khare A and Sukhatme U 1995 Phys. Rep. 251267

[5] Szegö G 1967 Orthogonal Polynomials (Am. Math. Soc. Colloquium Publications 23, 3rd ed.) pp 16-18; Zwillinger D 1992 Handbook of Differential Equations (Academic Press, 2nd ed.) Chapter 32

[6] Dutt R, Gangopadhyaya A and Sukhatme U P 1997 Am. J. Phys. 65400

[7] Gradshteyn I S and Ryzhik I M 1965 Table of Integrals, Series and Products (New York: Academic Press) p 43

[8] Infeld L and Hull T E 1951 Rev. Mod. Phys. 23 21; See also, Humi M 1968 J. Math. Phys. 91258

[9] Dutt R, Khare A and Sukhatme U P 1988 Am. J. Phys. 56163

[10] Villalba V M 1994 Phys. Lett. A 193218

[11] Wang Z X and Guo D R 1989 Special Functions (Singapore: World Scientific) Exercise 47 at p 291

[12] Avery J 1989 Hyperspherical Harmonics: Applications in Quantum Theory (Dordrecht: Kluwer Academic Publishers) 